American Journal of Applied Sciences 9 (1): 7-12, 2012

ISSN 1546-9239

(C) 2012 Science Publications

\title{
Extra-Bone Marrow Sites of Haemopoeisis: Dihydroartemisinin Effects in Wistar Albino Rats
}

\author{
${ }^{1}$ Nedosa Uchechukwu Anastasia, ${ }^{2}$ Saleh Mohammed, \\ ${ }^{3}$ Nedosa Kenechi Stanislaus, ${ }^{4}$ Njoku Goddy, \\ ${ }^{5}$ Ikenna Kenneth Onyedibe, ${ }^{6}$ P.A. Akah and ${ }^{6}$ C.O. Okoli \\ ${ }^{1}$ Department of Pharmacology and Toxicology, Faculty of Pharmaceutical Sciences, \\ Nnamdi Azikiwe University, P.M.B. 5025, Awka, Anambra State, Nigeria \\ ${ }^{2}$ Department of Biochemistry Federal, \\ College of Veterinary and Medical Laboratory Technology, \\ Vom, Plateau State, Nigeria \\ ${ }^{3}$ Evangelical Churches of West Africa (ECWA) Hospital, \\ Egbe, Kogi State, Nigeria \\ ${ }^{4}$ Medical Instructional Technology Unit, \\ Jos University Teaching hospital, \\ University of Jos, P.M.B. 2076, Jos, Plateau State, Nigeria \\ ${ }^{5}$ Department of Medical Microbiology, Jos University Teaching Hospital, \\ University of Jos, P.M.B. 2076, Jos, Plateau State, Nigeria \\ ${ }^{6}$ Department of Pharmacology and Toxicology, University of Nigeria, \\ Nsukka, Enugu State, Nigeria
}

\begin{abstract}
Problem statement: Iron is present in ferritin, the storage form of iron in the tissues; in the respiratory cytochrome enzymes; in hemoglobin of the blood and in the myoglobin of muscle Dihydroartemisinin (DHA) has been shown to interact with heme groups in vivo and in vitro. This study investigated the effects of 5 day and 7day oral dihydroartemisinin treatments on the blood and tissues of the lungs, the heart, the liver, the intestines, the spleen and the kidney of Wistar albino rats. The dosage regimens of dihydroartemisinin employed in the study were: A single dosage regimen of 1 $\mathrm{mg} \mathrm{kg}$; a repeated dosage regimen of 1; 2; 60 and $80 \mathrm{mg} \mathrm{kg}^{-1}$. Approach: The results of the study showed that dihydroartemisinin interacted with the hemoglobin of the blood and the myoglobin of muscle to stimulate new haemopoesis in a concentration, repetition and time dependent manner in the tissues of the lungs, liver, spleen, intestine, heart and kidney of Wistar albino rats which was absent in the control rats. Results: Statistically significant increases were observed in the Packed Cell Volume (PCV) $(\mathrm{P}<0.01-03)$ and the total White blood cell count $(\mathrm{P}<0.01)$ of the DHA-treated rats but not in the control rats. It also stimulated hyperplasia of erythrocyte and leucocyte stem cells in the lungs, liver, intestine, spleen, heart and kidney of DHA-treated Wistar albino rats not seen in the controls. Conclusion: These haemopoetic effects of DHA were greater and of longer duration in 5 day DHAtreatment rats than in those of the 7 day DHA-treatment rats.
\end{abstract}

Key words: Dihydroartemisinin (DHA), bone marrow, wistar albino rats, haemopoetic effects, dosage regimen, blood cells, Packed Cell Volume (PCV), Blood Cell Count (WBC)

\section{INTRODUCTION}

The activity of artemisinin and synthetic endoperoxides is related to their interaction with heme And the potentiating of artemisinin in vitro and in vivo by natural and synthetic heme models has been reported.

Many studies have shown that artemisinin and dihydroartemisinin were active against many cell lines in vitro.

Corresponding Author: Utoh-Nedosa Uchechukwu Anastasia, Department of Pharmacology and Toxicology, Faculty of Pharmaceutical Sciences, Nnamdi Azikiwe University, P.M.B. 5025, Awka, Anambra State, Nigeria 
Artemisinin-tagged transferring was found to be highly selective and potent in killing cancer cells Cancer cells including drug-resistant cancer cells are more susceptible to artemisinin drugs under conditions of high iron availability Haem was demonstrated to cause a $0.6 \mathrm{~V}$ shift in the reduction potential of artemisinin Artemisinin forms adducts with haem which retain the haem structure and lose the artemisinin structure In vitro data suggest that artemisinin-haem adducts don't have much antimalarial activity

Our study investigated the effect of dihydroartemisinin on the blood and six organs of Wistar albino rats.

\section{MATERIALS AND METHODS}

Five rats which weighed 104-106 grams and 7590 grams were evaluated for the effects of four oral dosage regimens and a repeated dosage regimen of Dihydroartemisinin (DHA). Four rats of equivalent weights as the test rats were given orally administered distilled water to serve as controls in each experiment.

The tested doses of DHA were $1 \mathrm{mg} \mathrm{kg}^{-1}$ DHA; a repeated dosage regimen of $1 ; 2 ; 60$ and $80 \mathrm{mg} \mathrm{kg}^{-1}$ DHA.

The rats which weighed $75-90$ grams received $1 \mathrm{mg}$ $\mathrm{kg}^{-1}$ rat weight of DHA for 5 or 7 days, rested for one week and received the same dosage regimen again for 5days or 7 days. The rats which weighed 104-106gms received the $1,2,60$ or the $80 \mathrm{mg} \mathrm{kg}^{-1}$ rat weight of DHA regimen for 5 or 7 days.

Blood samples from the test and control rats were collected from the subclavian artery $24 \mathrm{~h}$ after the administration of the last dose of0 each dosage regimen of DHA tested into blood collection bottles which contained EDTA anti-coagulant. The heart; liver; lungs; intestine (strip); spleen; and kidney of the sacrificed test and control rats were collected after gross anatomical observations of the organs in situ.

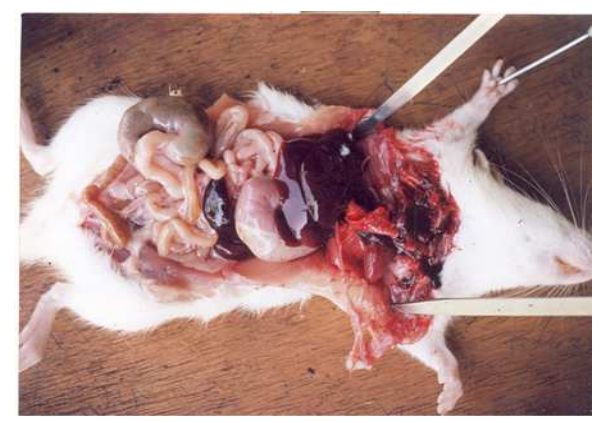

(a)
Photomicrographs of the tissues of these organs were prepared using conventional methods. The Packed Cell Volume (PCV) and the total white blood cell count of the blood of the test and control rats were determined using conventional haematological analytical methods.

\section{RESULTS}

Gross anatomical examination of the dihydroartemisinin-treated and control rats showed that there was blood congestion (increased population of the blood cells) in the lungs, liver, heart, spleen and kidney of DHA-treated rats but not in the control rats (Fig. 1) which is an evidence of new haemopoesis in the DHA-treated rats.

Comparism of the haematological data on the blood of the DHA-treated rats with those of the controls showed that dihydroartemisinin treatment produced statistically significant $\quad(\mathrm{P}<0.01-03)$ increases in the packed cell volume (PCV) of the DHA-treated rats which was absent in the controls. Dihydroartemisinin treatment also produced large significant increases in the white Blood Cell Count (WBC) $(\mathrm{P}<0.01)$ of the DHA-treated rats.

The PCV of the blood of rats treated for 5 days with DHA ranged from $45-48 \%$ while those of rats treated for 7days with DHA ranged from 30-39\% against a normal of $42 \%$. The red blood cells from 5 day DHA-treated rats were normochromic and normocytic ; had polychromasia and contained many reticlocytes. Some red blood cells of the blood from 7 day DHA-treated rats were normocytic and normochromic while the rest were mildly hypochromic and normocytic.

The WBC counts of the 5-day DHA treatment rats ranged from $10500-13500 \mathrm{~mm}^{3}$ while those of the 7 day treatments ranged from $3250-7050 \mathrm{~mm}^{3}$ (against a WBC count of $4800-5200 \mathrm{~mm}^{3}$ of the controls).

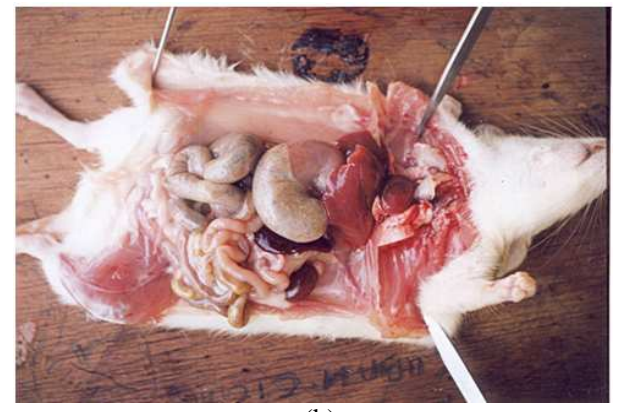

(b)

Fig. 1: Drk-red engorgement of the heart, lungs, liver, intestine, spleen and kidney of DHA-treated rat (a) absent in the control rat (b) under the influence of oral DHA illustrates these organs as haemopoeitic sites in the body 
Dose, repetition and time dependent hyperplasia of dark-red stained clustered cells in muscle of DHA tested organs which were absent in the controls were interpreted as histological evidence of new haemopoetic activity in the lungs, liver, heart, intestine, spleen and the kidney (Fig. 2-7). The $2 \mathrm{mg}$ $\mathrm{kg}^{-1}$ dose of DHA produced the maximal response for both the blood and tissue haemopoetic effects of DHA.

These evidences of new haemopoesis seen in the tissue micrographs of the lungs, heart, intestine, liver, spleen and kidney of the DHA-treated rats but not in those of control rats were deduced as indications that these six organs are extra bone marrow sites of haemopoesis.

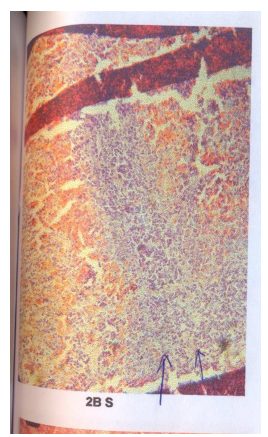

(a)

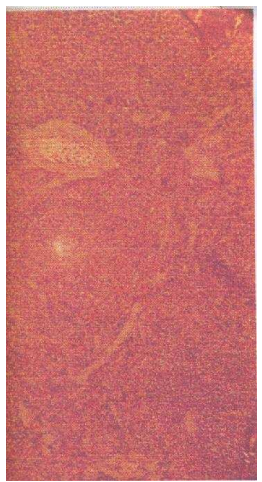

(b)

Fig. 2: The dark heavy staining of sites of new multiplication of cells in the photomicrograph of DHA-treated rat spleen (a) which are absent in that of controls (b) indicate new haemopoeisis in the DHA- treared rats

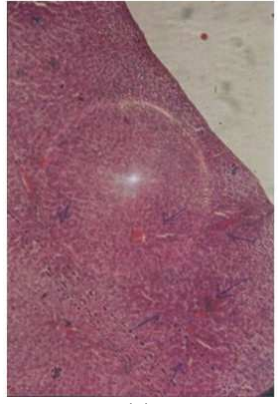

(a)

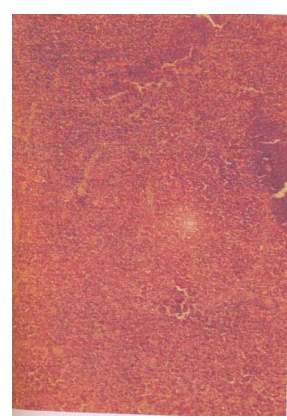

(b)

Fig. 3: Dark-stained sites containing large numbers of nuclei of cells at sites of new haemopoeisis in DHA-treated rat liver (a) which are absent in that of the control (b) show that DHA treatment produced new haemopoesis in the test rats

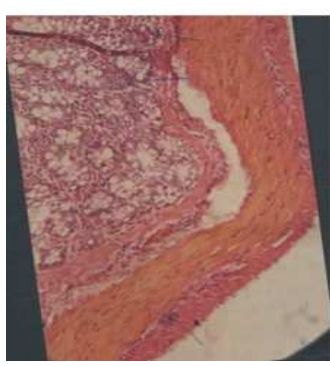

(a)

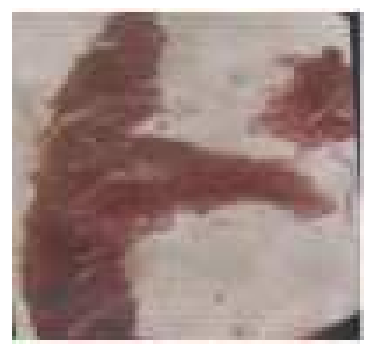

(b)

Fig. 4: Dark red stained proliferated cells seen in photomicrographs of DHA-treated rat (a) but not in that of a control rat (b), indicated that DHA stimulated new haemopoeisis in the test rats 


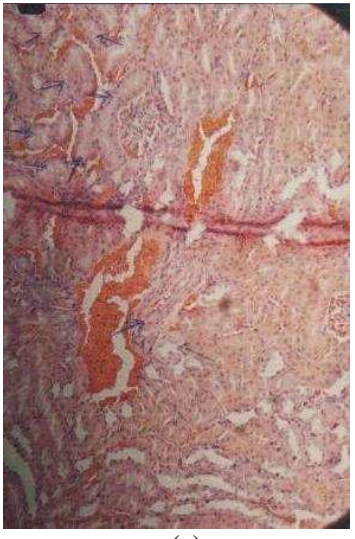

(a)

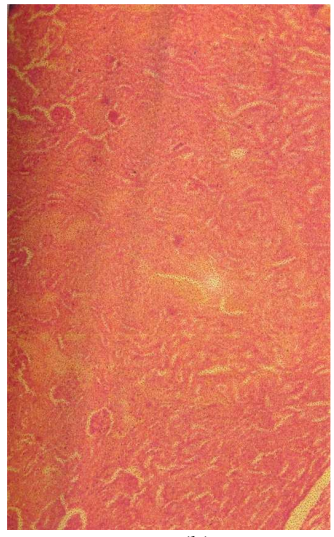

(b)

Fig. 5: Dark red heavily stained sites which contain many cells which are present in the photomicrographs of the kidney of DHA-treated rat (a) and absent in those of control rat (b) indicate that DHA stimulated new haemopoeisis in the kidney of the test rats

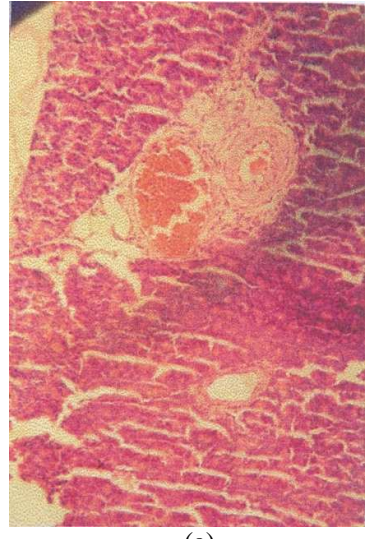

(a)

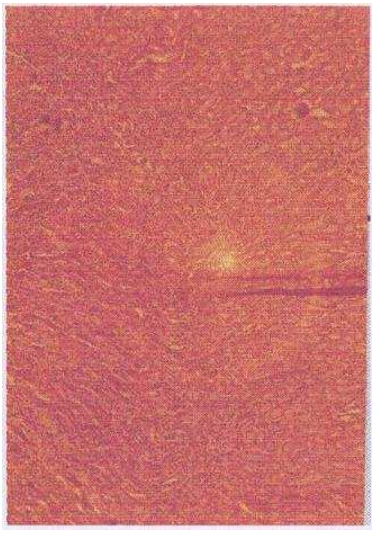

(b)

Fig. 6: Dark red stained proliferated cells seen in photomicrographs of DHA-treated rat spleen (a) which were absent in that of a control rat (b) shows that DHA treatment produced new haemopoeisis in the heart of the test rats

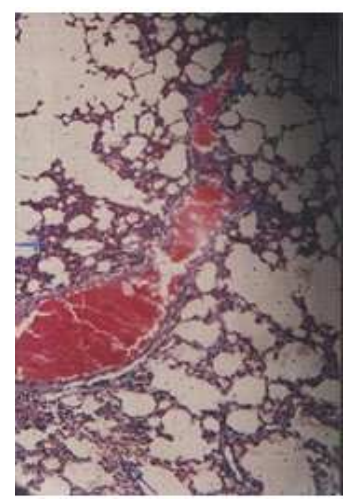

(a)

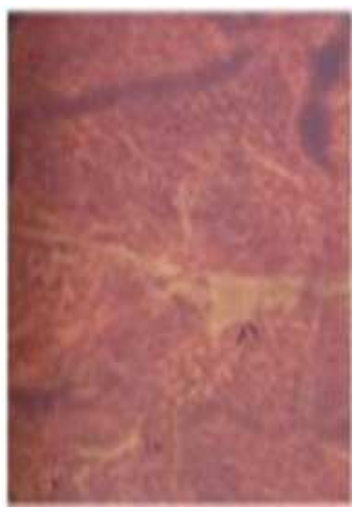

(b)

Fig. 7: Dark stained multiplied blood cells seen in Photomicrograph Of the lungs of DHA-treated rat (a) and that of control rat (b) which show that DHA produced haemopoeisis in the DHA-treated rats 
Figure 1 a shows dark red coloration of the heart, lungs, liver, spleen and kidney of DHA-treated rats in which DHA caused new haemopoiesis. Figure 1b shows equivalent organs of a control rat.

The erythropoietic sites stimulated by DHA to produce the increases in the bood seen in the gross anatomical display in Fig. 1 are indicated by the pointing arrows in the micrograph of the kidney (Fig. 2a), the liver (Fig. 3a), the intestine (Fig. 4a) the heart (Fig. 5a); the spleen (Fig. 6a), the lungs (Fig. 7a). The equivalent micrographs of the kidney, liver, intestine, heart, spleen and lungs of control rats are shown in (Figs. 2-7b) respectively.

\section{DISCUSSION}

Some drugs have been shown to be able to protect blood cells from damage while other drugs have effectively managed cancer of the blood (Erer et al., 2000; Tuan et al., 1992; Limoli et al., 2003; Reiter et al., 1997; Vijayalaxmi et al., 1996; 1999; Easton, 1974; Chun et al., 1991; Lyytikainen et al., 1996). The multiplicity of substances which arrect the blood cells or the bone marrow show that a therapeutic agent like dihydroartemisinin that controls a disease of the blood [malaria can have profound effects on the haemopoeitic system. The statistically significant increases in the PCV $(\mathrm{P}<0.5)$ and the WBC $(\mathrm{P}<0.01)$ of the DHAtreated rats (and not in the control rats) show that DHA stimulated the production of large numbers of new red and white blood cells. The presence of large numbers of reticlocytes and left-shifted neutrophils in the blood of DHA-treated rats but not in those of controls rats suggests that new haemopoesis took place.

The dose; repetition and time dependent blood congestion observed gross anatomically and the hyperplasia of blood cells observed histologically, provide additional evidences of new haemopoesis in DHA- treated rats as these were absent in the controls.

From a number of studies, it was concluded that when artemisinin reacts with haemoproteins, it alkylates the protein portion and not the haem portion as there was no reaction in solution between globin and dihydroartemisinin but there was marked reaction between haemoglobin and dihydroartemisinin In this reaction most of the drug molecules bound to the protein (globin) and not to the haem The dihydroartemisinin-myoglobin complex and the dihydroartemisinin-globin complex produced the new haemopoiesis seen as DHA dose dependent dark red colorations of the tissue photomicrographs of the different organs of DHA treated rats (Fig. 2-7).
The results of our study suggest that the dihydroartemisinin-myoglobin complex resulted in the dose, repetition and time dependent stimulation, proliferation and maturation of the haemopoesis stem cells in the lungs, the liver, the intestines, the heart, the spleen and the kidney of the DHA-treated albino rats. The maturation of the haemopoetic stem cells resulted in the similarly dose, repetition and time dependent statistically significant increases in the packed cell volume and total white blood cell counts obtained in the DHA-treated rat but not in the controls.

\section{CONCLUSION}

The findings of this study show that dihydroartemisinin stimulated new haemopoesis in the lungs, the heart, the liver, the intestines, the spleen and the kidney of Wistar albino rats suggesting that each of these organs is an extra-bone marrow site of haemopoesis. The results of this study also illustrated why dihydroartemisinin effects are affected in vivo and in vitro by hermin, porphyrin and metalloporphyrins.

\section{REFERENCES}

Erer, B., M. Galimberti, G. Lucarelli, C. Giardini and P. Polchi et al., 2000. Trichosporon beigelii: A lifethreatening pathogen in immunocompromised hosts. Bone Marrow Transplant., 25: 745-749. PMID: 10745260

Chun, H.G., B. Leyland-Jones and B.D. Cheson, 1991. Fludarabine phosphate: A synthetic purine antimetabolite with significant activity against lymphoid malignancies. J. Clin. Oncol., 9: 175188. PMID: 1702143

Easton, J.E., 1974. Haemopoietic stem cells. CIBA foundation symposium 13 (New Series). Immunology, 26: 229-229.

Limoli, C.L., E. Giedzinski, W.F. Morgan, S.G. Swarts and G.D. Jones, 2003. Persistent oxidative stress in chromosomally unstable cells. Cancer Res., 63: 3107-3111. PMID: 12810636

Lyytikainen, O., T. Ruutu, I. Lautenschlaager, L. Volin and L. Jokipii et al., 1996. Late onset pneumocystis carinii pneumonia following allogeneic bone marrow transplantation. Bone Marrow Transplant., 17: 1057-1060.

Reiter, R., L. Tang, J.J. Garcia and A. Munoz-Hoyos, 1997. Pharmacological actions of melatonin in oxygen radical pathophysiology. Life Sci., 60: 2255-2271. DOI: 10.1016/S0024-3205(97)00030-1 
Tuan, I.Z., D. Dennison and D.J. Weisdorf, 1992. Pneumocystis carinii pneumonitis following bone marrow transplantation. Bone Marrow Transplant., 10: 267-72. PMID: 1422481

Vijayalaxmi, M.L. Meltz, R.J. Reiter and T.S. Herman, 1999. Melatonin and protection from genetic damage in blood and bone marrow: whole-body irradiation studies in mice. J. Pineal Res., 27: 221225. DOI: 10.1111/j.1600-079X.1999.tb00618.x
Vijayalaxmi, R.J. Reiter, T.S. Herman and M.L. Meltz, 1996. Melatonin and radioprotection from genetic damage: In vivo/in vitro studies with human volunteers. Mut. Res., 371: 221-228. DOI: 10.1016/S0165-1218(96)90110-X 